\title{
Análise do perfil hematológico e parasitológico de comunidades ribeirinhas da região amazônica
}

\author{
Analysis of the hematological and parasitological profile of riverside communities in the amazon \\ region
}

Analisis del perfil hematológico y parasitológico de las comunidades ribereñas en la región amazónica

Recebido: 04/03/2021 | Revisado: 11/03/2021 | Aceito: 15/03/2021 | Publicado: 21/03/2021

\author{
Gabriela Evelin Anjo Silva \\ ORCID: https//orcid.org/0000-0002-9055-1994 \\ Universidade Federal do Pará, Brasil \\ E-mail: gabrielae.anjo@gmail.com \\ Érica dos Santos Sarges \\ ORCID: https//orcid.org/0000-0003-0206-0168 \\ Universidade Federal do Pará, Brasil \\ E-mail: ericasarges_15@hotmail.com \\ Larissa Nunes da Cruz \\ ORCID: https//orcid.org/0000-0002-3999-2022 \\ Universidade Federal do Pará, Brasil \\ E-mail: larissacruz336@gmail.com \\ Cleidiane dos Santos Sarges \\ ORCID: https//orcid.org/0000-0001-8833-4803 \\ Universidade Federal do Pará, Brasil \\ E-mail: clesargessantos@gmail.com \\ Marly de Fátima Carvalho de Melo \\ ORCID: https//orcid.org/0000-0001-8370-0819 \\ Universidade Federal do Pará, Brasil \\ E-mail:mfcm@ufpa.br \\ Ana Cristina Lo Prete \\ ORCID: https//orcid.org/0000-0002-2874-5296 \\ Universidade São Judas Tadeu, Brasil \\ E-mail: analoprete@gmail.com \\ Carolina Heitmann Mares Azevedo Ribeiro \\ ORCID: https//orcid.org/0000-0002-9457-2733 \\ Universidade Federal do Pará, Brasil \\ E-mail: chribeiro@ufpa.br
}

\begin{abstract}
Resumo
Objetivo: Analisar a ocorrência e os determinantes de anemia e parasitose em moradores de comunidades fluviais da região amazônica. Métodos: Entre 2016 e 2017 foram coletadas amostras biológicas e dados socioeconômicos, alimentares e sanitários de 213 indivíduos residentes nas comunidades de Maracujá e Murucutum. Foram realizadas análises hematológicas da contagem total de eritrócitos, hemoglobina, hematócrito, VCM (Volume Corpuscular Médio), HCM (Hemoglobina Corpuscular Média), CHCM (Concentração da Hemoglobina Corpuscular Média), RDW (Redcell volume Distribution Width), leucócitos totais e plaquetas. Amostras de fezes foram analisadas para ivestigação de helmintos e protozoários. Resultados: Dos 213 sujeitos, apenas 4,70\% (10) não apresentaram nenhuma das condições estudadas. Na análise hematológica, $22 \%(\mathrm{n}=47)$ apresentaram anemia, com 23 do tipo microcítica e hipocrômica e 24 normocítica e normocrômica. Estavam infectados por por parasitas intestinais $93 \%$ (n=198) dos sujeitos estudados. Na análise da associação entre ocorrência de parasitose e anemia, 19,71\% (42) indivíduos apresentaram ambas as condições. Conclusão: A alimentação baseada em açaí, carne bovina e de peixe proporciona à população estudada uma proteção contra anemias de origem alimentar, enquanto são altas as ocorrências de parasitoses e anemias associadas a estas devido às precárias condições de higiene e saneamento básico nessa população.
\end{abstract}

Palavras-chave: Anemia; Parasitoses; Comunidade ribeirinha.

\section{Abstract}

Objective: analyze the occurrence and determinants of anemia and parasitosis in residents of river communities in the Amazon region. Methods: Between 2016 and 2017, biological samples and socioeconomic, food and health data were 
collected from 213 individuals living in the communities of Maracujá and Murucutum. Hematological analyzes of the total erythrocyte count, hemoglobin, hematocrit, VCM (Mean Corpuscular Volume), HCM (Mean Corpuscular Hemoglobin), CHCM (Mean Corpuscular Hemoglobin Concentration), RDW (Redcell volume Distribution Width), total leukocytes and platelets were performed. Stool samples were analyzed for investigation of helminths and protozoa. Results: Of the 213 subjects, only $4.70 \%$ (10) did not present any of the studied conditions. In the hematological analysis, 22\% (47) presented anemia, with 23 microcytic and hypochromic and 24 normocytic and normochromic. 93\% (198) of the studied subjects were infected with intestinal parasites. In the analysis of the association between the occurrence of parasitosis and anemia, 19.71\% (42) individuals had both conditions. Conclusion: The diet based on açaí, beef and fish provides the studied population with protection against food-borne anemias, while parasites and anemias associated with these are high due to the precarious conditions of hygiene and basic sanitation in this population.

Keywords: Anemia; Parasitoses; Riverside community.

\section{Resumen}

Objetivo: Analizar la ocurrencia y determinantes de anemia y parasitosis en pobladores de comunidades fluviales de la región amazónica. Métodos: Entre 2016 y 2017 se recolectaron muestras biológicas y datos socioeconómicos, alimentarios y de salud de 213 individuos que habitan en las comunidades de Maracujá y Murucutum. Se realizaron análisis hematológicos del recuento total de eritrocitos, hemoglobina, hematocrito, VCM, HCM, CHCM, RDW, leucocitos totales y plaquetas. Se analizaron muestras de heces para la investigación de helmintos y protozoos. Resultados: De los 213 sujetos, solo el 4.70\% no presentó ninguna de las condiciones estudiadas. En el análisis hematológico, el $22 \%$ presentó anemia, con 23 microcíticas e hipocrómicas y 24 normocíticas y normocrómicas. El 93\% de los sujetos estudiados estaban infectados con parásitos intestinales. En el análisis de la asociación entre la ocurrencia de parasitosis y anemia, el 19,71\% individuos tenían ambas condiciones. Conclusión: La dieta a base de açaí, carne y pescado brinda protección a la población estudiada frente a las anemias de origen alimentario, mientras que la ocurrencia de parásitos y anemias asociadas a estas es alta debido a las precarias condiciones de higiene y saneamiento básico de esta población.

Palabras clave: Anemia; Parasitosis; Comunidad riberenã.

\section{Introdução}

As comunidades ribeirinhas da Amazônia, prevalentes na região norte do Brasil, são populações residentes às margens de rios. Devido à alta prevalência, é importante estudar a influência das formas de moradia, alimentação e saúde nessas populações (Catarina, et al., 2005), uma vez que a ausência de políticas públicas voltadas para um adequado saneamento básico, assim como de assistência às condições higiênicas e alimentares nessas populações (Santos \& Merlini, 2010), contribuem para uma má qualidade de vida, com consequente maior predisposição a anemias de origem alimentar e/ou parasitária nessas populações.

A anemia ocorre quando há alteração no tamanho dos eritrócitos e quando o índice da hemoglobina contida nos mesmos está abaixo dos valores considerados normais (Lima, 2007). Esta condição pode ser causada por vários fatores, sendo o mais comum deles, a deficiência de ferro, geralmente como consequência de uma carência alimentar, acometendo principalmente crianças, mulheres em idade fértil e gestantes (Stoltzfus \& Dreyfuss, 2013)._No entanto, outro fator determinante para o desenvolvimento de anemia é a ocorrência de parasitas intestinais.

Os parasitas podem causar anemia ferropriva por reduzir em até $20 \%$ o ferro ingerido na dieta (WHO, 2001). Os parasitas mais associados à anemia ferropriva são ancilostomídeos (Ancylostoma duodenale e Necator americanus), Trichuris trichiura (Souza, et al., 2002), Entamoeba histolytca e Endolimax nana, embora Ascaris lumbricoides e da Giardia lamblia também possam causar esta condição (Walcher \& Frizzo, 2013). Além disso, tanto helmintos quanto protozoários podem causar anemia pela espoliação do sangue ou por hemorragia causada pela sua presença.

Os moradores das comunidades Furo do Maracujá e Murucutum, que ficam localizadas próximas ao município de Belém do Pará, são exemplos de ribeirinhos que estão propensos a parasitoses e anemias. Diversas condições de vida em que estão expostos, tais como saneamento básico inadequado, tratamento de água ineficaz e até mesmo inexistente e $_{2}$ uma atenção básica à saúde incompleta e restrita (Chieffi et al., 1982) aumentam os riscos de parasitose nessas populações, assim como de 
anemias resultantes desses quadros. Por outro lado, estes ribeirinhos, em sua grande maioria, consomem principalmente alimentos ricos em proteínas, minerais e vitaminas, como o açaí e o peixe, que enriquecem nutricionalmente a alimentação dessas populações (Murrieta, et al., 2008), o que pode alterar o padrão de ocorrência e/ou agravamento de anemia de origem alimentar, em especial a ferropriva, em algumas populações ribeirinhas.

Desse modo, para conhecer a realidade da saúde dessas populações que residem nas margens dos rios da Amazônia e melhorar a assistência às mesmas, o objetivo do estudo foi avaliar a ocorrência e determinantes de parasitoses e anemia em moradores dessas duas comunidades ribeirinhas.

\section{Métodos}

Trata-se de um estudo transversal, de natureza quantitativa realizado nos anos de 2016 e 2017, nas comunidades ribeirinhas Furo do Maracujá e Murutucum, situadas na área insular do Estado do Pará,_atendidas pelo projeto Luz da Amazônia.

Este trabalho foi aprovado pelo Comitê de Ética em Pesquisa com seres humanos (1.351.214). Todas as análises foram realizadas apenas nos indivíduos que aceitaram participar da pesquisa e assinaram o termo de compromisso livre e esclarecido (TCLE). No caso dos sujeitos menores de 18 anos, o responsável legal assinou.

Foi estudada uma população de 213 ribeirinhos residentes em uma das comunidades estudadas, constituída por crianças e adultos de 1 a 60 anos, de ambos os sexos, reunidos em 163 núcleos familiares.

Os participantes foram divididos em faixas etárias de acordo com a Organização Mundial de Saúde (OMS) para classificação de anemia. Para análise da ocorrência de anemia e parasitoses, assim como a associação entre as duas condições, foram incluídos no estudo os sujeitos residentes de uma das duas comunidades ribeirinha estudadas e que realizaram ambos os exames, hematológico e parasitológico. Foram excluídos do estudo os sujeitos que realizaram apenas um dos exames ou que a amostra tenha sido comprometida para a análise.

Os dados sobre renda familiar, tipo de obtenção de água ingerida, esgotamento sanitário e padrão de consumo alimentar foram obtidos por meio de questionários aplicados para cada chefe da família. No caso do padrão alimentar, foi avaliada a porcentagem de famílias que apresentavam o consumo frequente (mais de 3 vezes na semana) de cada um dos alimentos listados: açaí, demais frutas (sem distinção), carne de origem bonina, de frango e de peixe, legumes (sem distinção) e feijão.

A amostra de sangue para realização do hemograma foi colhida por punção venosa em tubo contendo EDTA como anticoagulante. As mesmas foram analisadas por meio de metodologia semiautomatizada, sendo avaliados os seguintes parâmetros de contagem total de eritrócitos, hemoglobina, hematócrito, VCM (Volume Corpuscular Médio), HCM (Hemoglobina Corpuscular Média), CHCM (Concentração da Hemoglobina Corpuscular Média), RDW (Redcell volume Distribution Width), leucócitos totais e plaquetas. Para visualização de possíveis alterações celulares, como poiquilocitoses, a extensão sanguínea foi realizada em todas as amostras e submetidas à microscopia óptica como método para a visualização dos elementos celulares.

Para investigação da ocorrência de parasitoses intestinais, foi coletada uma amostra de fezes de cada indivíduo. As amostras foram analisadas pelos métodos de Faust e o de Hoffmann, Pons e Janer, utilizados para detecção de helmintos e protozoários (Hoffman, et al., 1934).

As análises estatísticas sobre a ocorrência de anemia e parasitose na população foram realizadas no programa BioEstat 5.0. Entre os testes, o Odds Ratio foi feito para correlacionar a anemia e seus determinantes, entre eles a parasitose e padrão alimentar, e o teste de regressão logística simples, para determinar se a anemia era significativa em um sexo específico. 


\section{Resultados}

Dos 213 pacientes estudados, compreendidos em 163 famílias, 137 (64,3\%) pertenciam ao sexo feminino e 76 $(35,7 \%)$ ao sexo masculino. Na avaliação por faixa etária, 5,63\% (n=12) tinham idade entre 1 e 4 anos, 28,16\% (n=60) entre 5 e 11 anos, 8,45\% (n=18) entre 12 e 14 anos e $57,74 \%(n=123)$ entre 15 e 60 anos.

Os dados sobre saneamento básico e condição socioeconômica foram avaliados a partir dos 163 questionários, em que cada questionário representa uma família, e estão apresentados na Tabela 1. Algumas famílias apresentaram mais de uma resposta para a mesma pergunta.

Tabela 1: Dados socioeconômicos, ambientais e higiênicos das 163 famílias. Belém-PA, 2018.

\begin{tabular}{|c|c|c|}
\hline VARIÁVEIS & $N$ & $\%$ \\
\hline \multicolumn{3}{|l|}{ Condição socioeconômica } \\
\hline Menos de 1 salário mínimo & 111 & 66,0 \\
\hline 1 salário mínimo & 46 & 31,0 \\
\hline Acima de 1 salário mínimo & 6 & 3,0 \\
\hline \multicolumn{3}{|c|}{ Forma de obtenção da água para consumo } \\
\hline Direto do rio & 7 & 4,4 \\
\hline Água mineral & 7 & 4,1 \\
\hline Água mineral e poço & 36 & 3,7 \\
\hline Direto do poço & 143 & 87,8 \\
\hline \multicolumn{3}{|l|}{ Tratamento da água } \\
\hline Tratada com hipoclorito de sódio & 75 & 46,0 \\
\hline Nenhum tratamento & 88 & 54,0 \\
\hline \multicolumn{3}{|l|}{ Esgotamento sanitário } \\
\hline Fossa & 55 & 33,7 \\
\hline Sintina & 26 & 16,0 \\
\hline Nenhum tratamento & 121 & 74,3 \\
\hline
\end{tabular}

Fonte: Silva, et al (2021),

Na Tabela 2 é apresentado o padrão alimentar das famílias. Os resultados são em relação à porcentagem de famílias que relatam consumir frequentemente esses alimentos em suas refeições, sendo o alimento mais consumido entre a população estudada, presente em $81,12 \%$ das famílias, o açaí. 
Tabela 2: Padrão alimentar das famílias estudadas nas comunidades ribeirinhas. Belém-PA, 2018.

\begin{tabular}{|c|c|}
\hline Principais alimentos consumidos & $\%$ \\
\hline Açaí & $87,12 \%$ \\
\hline Carne bovina & $68,71 \%$ \\
\hline Peixe & $51,53 \%$ \\
\hline Frango & $43,56 \%$ \\
\hline Legumes (sem distinção) & $33,74 \%$ \\
\hline Feijão & $33,13 \%$ \\
\hline Demais frutas (sem distinção) & $33,52 \%$ \\
\hline
\end{tabular}

Fonte: Silva, et al (2021),

Em relação à avaliação do perfil hematológico dos 213 indivíduos estudados, apresentada na Tabela 3, 78\% (166) não apresentaram alterações hematológicas e 22\% $(\mathrm{n}=47)$ apresentaram anemia. Dos pacientes com anemia, 49\% $(\mathrm{n}=23)$ apresentaram anemia do tipo microcítica e hipocrômica e $51 \%(\mathrm{n}=24)$ do tipo normocítica e normocrômica.

Tabela 3: Índices hematimétricos dos indivíduos estudados nas comunidades ribeirinhas. Belém-PA, 2018.

\begin{tabular}{|c|c|c|}
\hline $\begin{array}{c}\text { Índices Hematimétricos (unidade de } \\
\text { medida) }\end{array}$ & Não anêmicos $(\mathbf{M} \pm \mathbf{D P})$ & Anêmicos $(\mathbf{M} \pm \mathbf{D P})$ \\
\hline Contagem de eritrócitos $\left(\times 10^{6} / \mathrm{mm}^{3}\right)$ & $4,6 \pm 0,32$ & $4,1 \pm 0,19$ \\
\hline Hemoglobina (g/dL) & $13,3 \pm 0,93$ & $11,4 \pm 0,53$ \\
\hline Hematócrito (\%) & $40,1 \pm 3,12$ & $34,5 \pm 1,35$ \\
\hline VCM (fL) & $85,7 \pm 6,99$ & $80,2 \pm 8,07$ \\
\hline HCM (pg) & $29 \pm 2,43$ & $26,46 \pm 1,41$ \\
\hline CHCM (\%) & $33,01 \pm 0,90$ & $32,77 \pm 0,53$ \\
\hline RDW (\%) & $15,4 \pm 3,3$ & $15,1 \pm 3,68$ \\
\hline
\end{tabular}

Legenda. M: média; DP: desvio padrão; $\mathrm{mm}^{3}$ : milímetro cúbico; g/dL: grama por decilitro; \%: porcentagem; pg: picograma; fL: fentolitro. Fonte: Silva, et al (2021),

A caracterização de casos de anemia em leve, moderada e severa foi feita com base classificação da Organização Mundial da Saúde ${ }^{7}$ e avaliada em separado nas diferentes faixas etárias (Tabela 4), e sexo, no caso dos sujeitos acima de 15 anos (Tabela 5).

Tabela 4: Caracterização do grau de anemia por faixa etária. Belém-PA, 2018.

\begin{tabular}{lccc}
\hline \multicolumn{4}{c}{ ANEMIA } \\
\hline \multicolumn{1}{c}{ Faixa etária } & Leve & Moderada & Severa \\
\hline $1-4$ anos & $83,33 \%(\mathrm{n}=5)$ & $16,66 \%(\mathrm{n}=1)$ & $0 \%(\mathrm{n}=0)$ \\
$5-11$ anos & $83,33 \%(\mathrm{n}=10)$ & $16,66 \%(\mathrm{n}=2)$ & $0 \%(\mathrm{n}=0)$ \\
$12-14$ anos & $66,66 \%(\mathrm{n}=2)$ & $33,33 \%(\mathrm{n}=1)$ & $0 \%(\mathrm{n}=0)$ \\
$15-60$ anos & $88,46 \%(\mathrm{n}=23)$ & $7,69 \%(\mathrm{n}=2)$ & $3,84 \%(\mathrm{n}=1)$ \\
\hline
\end{tabular}

Legenda: os dados são expressos em porcentagem (\%) e número de sujeitos (n). Fonte: Silva, et al (2021),

Na avaliação da ocorrência e gravidade das anemias dos indivíduos na faixa etária de 15 a 60 anos, foi observado que no sexo masculino houve anemia em 6 sujeitos, todos casos leves, enquanto no sexo feminino houve 20 ocorrências de 
anemia, com casos leves, moderados e um caso severo, como mostra a tabela 5. No entanto, não foi observada correlação entre a ocorrência de anemia e o sexo do sujeito $(\mathrm{p}=0,7901)$.

Tabela 5: Caracterização do grau de anemia por gênero dos indivíduos acima de 15 anos. Belém-PA, 2018.

\begin{tabular}{llll}
\hline \multicolumn{4}{c}{ ANEMIA } \\
\hline Sexo & \multicolumn{1}{c}{ Leve } & Moderada & Severa \\
\hline Feminino & $85 \%(\mathrm{n}=17)$ & $10 \%(\mathrm{n}=2)$ & $5 \%(\mathrm{n}=1)$ \\
Masculino & $100 \%(6)$ & $0 \%(\mathrm{n}=0)$ & $0 \%(\mathrm{n}=0)$ \\
\hline
\end{tabular}

Fonte: Autores.

Na avaliação do perfil parasitológico, dos 213 pacientes estudados, 93\% $(n=198)$ estavam infectados por parasitas intestinais. As espécies mais prevalentes foram Trichuris trichiura (47\%), Ascaris lumbricóides (27\%), Ancilostomídeo (19\%), Giardia lamblia (6\%) Entamoeba coli (21\%) e Endolimax nana (49\%).

$\mathrm{Na}$ análise da associação entre ocorrência de parasitose e anemia, 19,71\% (42) indivíduos apresentaram a associação, 73,24\% (156) apresentaram apenas parasitoses, 2,35\% (5) apresentaram apenas quadro anêmico e 4,70\% (10) não apresentaram nenhuma das condições. O teste foi estatisticamente significativo (OR de 131,04; IC95\%: 42,48-404,16; p-valor = 0,0001). O número necessário para causar um evento desfavorável (NNH) foi igual a 2, o que significa que são necessários dois indivíduos expostos a parasitoses para que um indivíduo apresente anemia.

$\mathrm{Na}$ avaliação dos pacientes que apresentaram ambas as condições, anemia e parasita intestinal, a frequência dessa relação para cada um dos parasitas encontrados está apresentada na Figura 1, tendo sido a parasitose mais prevalentemente associada à anemia a causada por Trichuris trichiura.

Figura 1: Frequência das espécies parasitológicas em pacientes anêmicos. Belém-PA, 2018.

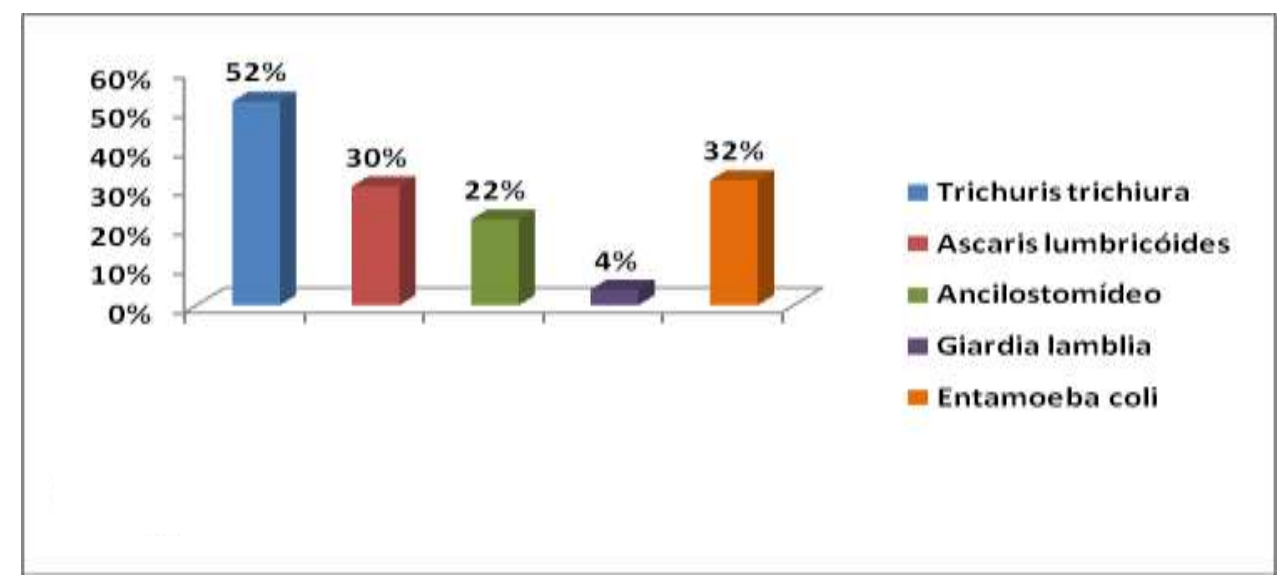

Fonte: Silva, et al (2021),

\section{Discussão}

Segundo o Ministério da Saúde, o conceito de estado nutricional é definido como o equilíbrio entre o consumo de nutrientes e o gasto de energia do organismo para atender as necessidades nutricionais (Souza EB, 2010). Para manter esse equilíbrio, sugere-se uma dieta variada, o que não acontece com as duas populações estudadas, as quais consomem uma variedade restrita de alimentos.

A população do presente trabalho apresentou uma dieta rica principalmente em alimentos como o açaí, carne e peixe, o que pode explicar a baixa incidência de anemia_alimentar. 
A carne bovina é um alimento que possui alto valor nutricional, rico em gordura, vitaminas do complexo B (cobalamina, a niacina e a riboflavina), ferro, zinco (Young et al., 2013), proteínas e ácidos graxos. A presença da carne na dieta, é de grande importância, visto que, atende as necessidades da ingestão de ferro, auxiliando na prevenção de anemia (Medeiros, 2008).

Estudo como de Portinho e colaboradores. (2012) relata as diversas propriedades alimentares do açaí, em decorrência de sua composição de proteínas, fibras, lipídios, vitaminas (C, E, B1 e B2) e minerais (fósforo, ferro e cálcio). Esse alimento, além de possuir efeitos anti-inflamatórios, atuar no perfil imunológico e na prevenção de dislipidemias, diabetes tipo 2, síndrome metabólica, câncer e do próprio envelhecimento, atua também como preventivo para o desenvolvimento da anemia ferropriva. $\mathrm{O}$ ferro presente no açaí se encontra na forma não heme (menor absorvida no organismo), porém, quando aliado a outros componentes alimentares, como as vitaminas A e C, tem sua absorção aumentada (Menezes, et al., 2008).

Além do açaí, as carnes da dieta, que no caso da população estudada se tratava preferencialmente de carne bovina e carne de peixe, possuem ferro heme e uma variedade de nutrientes que auxiliam na absorção ou biodisponibilidade do ferro não heme, como é o caso de vitamina C. Além disso, também apresentam em sua composição nutrientes importantes para se evitar outros tipos de anemias carenciais, como a vitamina B12 (Whaley, et al, 2003). Assim como no presente estudo, outra pesquisa também observou que a ingestão de fontes de vitamina $\mathrm{C}$, feijões, carnes, açaí e peixes em crianças de baixo nível socioeconômico foi um fator de proteção na ocorrência de anemia ferropriva (Bortolini \& Fisberg, 2010).

Desse modo, a presença considerável do açaí e da carne de peixe na dieta da população estudada pode explicar o fato da maioria dos indivíduos não apresentarem anemia, muitas vezes mesmo na presença de parasitas intestinais, indicando que a quantidade de sangue que o parasita consome do organismo não é suficiente para causar nenhum dano ao paciente, devido às grandes quantidades ingeridas de ferro.

Por outro lado, quando avaliada a incidência de anemias em relação à faixa etária, foi observado uma elevação em crianças (1 a 11 anos), quando em comparação aos adolescentes e adultos, a qual pode ser explicada em virtude de nesta faixa etária haver elevada necessidade de ferro para repor as perdas energéticas, pois estão em desenvolvimento de expansão da massa celular vermelha e crescimento tecidual (Garanito et al., 2010), além dos hábitos de higiene serem geralmente mais precários, o que favorece a ocorrência de parasitoses. Vale ressaltar aqui o prejuízo de anemia em crianças, uma vez que pode provocar dificuldades na aprendizagem da linguagem, distúrbios psicológicos e comportamentais, além de debilitar a defesa imunológica, facultando a ocorrência e/ou agravamento dessas doenças infecciosas (Olivares \& Walter, 2004).

O presente trabalho não evidenciou diferença estatística quando analisada a correlação entre anemia e sexo do sujeito ( $\mathrm{p}=0,7901$ ), o que foi avaliado na faixa entre 15 e 60 anos, uma vez que nessa faixa etária houve 92 mulheres, com 20 casos de anemia (21,7\%), e 31 homens, com 6 casos de anemia (19,4\%). Esta ocorrência semelhante provavelmente se deva ao fato de as famílias apresentarem um padrão de consumo alimentar, assim como dos demais fatores avaliados (origem da água de consumo, saneamento), muito semelhante entre os integrantes de uma mesma residência. Já em relação à gravidade, quadros moderados e graves, mesmo que de forma pouco frequente, foram observados apenas em mulheres, podendo ser talvez atribuído ao ciclo menstrual, embora este fator não tenha sido avaliado no presente estudo.

Diante do exposto, a ocorrência de anemia, observada principalmente em crianças, pode ser proveniente das precárias condições de higiene pessoal e de saneamento e tratamento da água evidenciadas na população estudada, uma vez que tais condições favorecem a ocorrência de parasitoses intestinais (Mendes, 2013), levando secundariamente à ocorrência de anemias. No presente estudo, a análise estatística demonstrou que os indivíduos que possuíam parasitose tiveram maior associação de anemia, de modo que, a cada duas pessoas infectadas, uma apresentou anemia. 
Sabe-se que a água e o solo são meios importantes de transmissão das parasitoses, o que explica a predominância de doenças parasitárias nos lugares sem saneamento básico e falta de tratamento de água (Busato et al., 2014; Santos et al., 2014). Desse modo, a alta ocorrência de parasitoses intestinas na população estudada (93\%) pode estar relacionada às variáveis que favorecem a transmissão e aquisição destes parasitas, como é o caso do alto índice de famílias que utilizam água e poço $(87,7 \%)$ com próximo a metade delas $(46,7 \%)$ sem fazer nenhum tipo de tratamento antes do consumo. Somado a isso, a grande maioria $(74,2 \%)$ das famílias também não possuem esgotamento sanitário, fazendo com que os dejetos contaminem o solo, que por ser arenoso e em ambiente de clima quente e úmido, constituem uma importante fonte de contaminação e reinfecção parasitária por favorece o ciclo biológico destes parasitas. Vale ressaltar que a baixa renda familiar observada no estudo, com 66\% vivendo com menos de 1 salário-mínimo, está também relacionada à essa falta de acesso a condições de moradia adequada, com consequente aumento da precariedade das condições sanitárias (Andre et al., 2018), evidenciada pela inexistência de fossa sanitária, aumentando o risco do contato da população com dejetos e águas contaminadas por parasitas.

O parasitismo por helmintos e protozoários também é um fator de risco a ser levado em consideração no desenvolvimento de anemia ou no agravamento, quando sua origem não tem natureza nutricional, por exemplo (Gama, et al., 2008). Outro fator importante é a determinação da carga parasitária, pois quanto maior a carga, maior a probabilidade do indivíduo em apresentar infecções. Estudos em crianças com déficit nutricional demonstram que quanto maior a carga parasitária, maior a gravidade da doença quando comparada em crianças com condições nutricionais normais (Gama, et al., 2008), o que sugere que a anemia seja o resultado da associação da presença de parasitas e deficiência nutricional.

No presente estudo, dos 47 indivíduos com anemia, 51\% apresentaram anemia do tipo normocítica e normocrômica e 49\% do tipo microcítica e hipocrômica, esta última muito associada a carência de ferro. Além disso, todos (100\%) apresentaram parasitemia positiva associada (presença de mais de um parasita), sugerindo que a baixa de ferro possa ter grande relação com o parasito, uma vez que a alimentação é rica em ferro. Essa suspeita é reforçada uma vez que a maioria dos anêmicos eram portadores e parasitoses que resultam em quadros anêmicos, com 52\% dos anêmicos parasitados apresentando infecção por Trichuris trichiura, capaz de lesionar a parede intestinal, consumindo aproximadamente $0,005 \mathrm{~mL}$ de sangue/dia, seguido pela ocorrência de Ascaris lumbricoides (30\%), que além de ter uma ação espoliativa, pode vir a causar reações tóxicas no hospedeiro, e de Ancilostomídeos (22\%), sendo estes causadores da anemia de forma direta, visto que eles comprometem a absorção do ferro pelo organismo (Santos Júnior, et al., 2016). Ressalta-se que

Desse modo, devido ao alto consumo de fontes alimentares ricas em ferro e ao fato de estes parasitas intestinais poderem reduzir a absorção do ferro ingerido da dieta em até 20\% (Cantos, Dutra, Koerich, 2003), supostamente as parasitoses foram as principais causas de anemias na população estudada.

\section{Conclusão}

A população estudada é desprovida de condições adequadas de higiene e saneamento básico, assim como socioeconômicas, que acarretam maior exposição à contaminação e reinfecção parasitária e, por consequência, anemias provenientes dessa condição. No entanto, mesmo na ausência de uma alimentação diversificada, a dieta baseada em açaí e carne bovina e de peixe proporciona à população estudada uma proteção contra anemias de origem alimentar. Estudos adicionais serão necessários para melhor compreensão da associação alimentar com a saúde dos ribeirinhos.

\section{Agradecimento e Financiamento}

Ao Programa Luz na Amazônia, à Pró reitoria de Extensão (Proex) e ao Laboratório de Parasitologia da Universidade Federal do Pará. 


\section{Referência}

Andre, H. P. et al. (2018). Indicadores de insegurança alimentar e nutricional associados à anemia ferropriva em crianças brasileiras: uma revisão sistemática. Ciência \& Saúde Coletiva, 23 (4).

Barros, M. B. A. et al. (2016). Social inequalities in health behaviors among Brazilian adults: National Health Survey. International Journal for Equity in Health, (15), 148

Bortolini, G. A. \& Fisberg, M. (2010). Orientação nutricional do paciente com deficiência de ferro. Revista Brasileira de Hematologia e Hemoterapia, 13, 32:105

Busato, M. A. et al. (2014). Relação de parasitoses intestinais com as condições de saneamento básico. Cienc Cuid Saúde, 13(2), $357-363$.

Cantos, A. C. et al. (2003). Iron deficiency anemia occurrence in patients with Enteroparasitosis. Health Rev, 5(10), 43-48.

Catarina, A. et al. (2005). Anemia e Consumo Alimentar De Gestantes Adolescentes. Revista de Nutrição, 32-227.

Chieffi, P. P. et al. (1982). Epidemiological aspects of enteroparasitosis in the State of Sao Paulo, Brazil. Revista Paulista de Medicina, 99, 6-34.

Gama, A. S. M. et al. (2009). Prevalência de anemia em populações infantis ribeirinhas enteroparasitadas no médio Solimões-AM. Revista Paraense de Medicina, 2 (23).

Garanito, M. P. et al. (2010). Deficiência de ferro na adolescência. Revista Brasileira de Hematologia e Hemoterapia. 32 , 45-48.

Gomes, R. et al., (2007). Por que os homens buscam menos os serviços de saúde do que as mulheres? As explicações de homens com baixa escolaridade e homens com superior. Caderno de Saúde Pública, 23(3), 565-574.

Hoffman, W. A. et al. (1934). The sedimentation concentration method in Schistosoma mansoni. PRJ Public Health Tropical Medicine, 9, $283-291$.

Levorato, C. D. et al. (2013). Factors associated with the demand for health services from a gender-relational perspective. Ciência \& Saúde Coletiva, 5(1), 1263-1274.

Lima, W.A. (2007). Anemia associada às parasitoses intestinais.RevistaConexão,1-12.

Mendes, A. A. R. (2012). Saúde escolar e educação integral: a relação entre as parasitoses intestinais e o desempenho escolar do aluno da Escola Municipal de Ensino Fundamental Roberto Turbay em Ariquemes-RO. Dissertação de mestrado, Universidade Federal de Rondônia, Porto Velho.

Embrapa. (2008). Valor nutricional da carne bovina e suas implicações para a saúde. Recuperado de https://www.embrapa.br/busca-de-publicacoes//publicacao/326880/valor-nutricional-da-carne-bovina-e-suas-implicacoes-para-a-saude-humana.

Menezes, S. E. M. et al. (2008). Valor nutricional da polpa de açaí (Euterpe oleracea Mart) liofilizada. Acta Amazonica, 38, 6-311.

Murrieta, R. S. S. et al. (2008). Consumo alimentar e ecologia de população ribeirinhas em dois ecossistemas amazônicos: um estudo comparativo. Revista de Nutrição, 21, 123-133.

Olivares, M., \& Walter, T. (2004). Causas y consecuencias de la deficiencia de hierro. Revista de Nutrição, $17,05-14$.

Portinho, A.J. et al. (2012). Efeitos Benéficos do Açaí. International Journal of Nutrology, 5 (1), 15-20.

Santos, A. A. et al. (2014). Frequência de parasitos intestinais na U. I. M. Professora Magnólia Hermínia Araújo do município de Caxias- MA. Rev. Humana. $1,95-113$.

Santos Júnior, J. G. A. et al. (2016). Anemia associada às parasitoses intestinais de pacientes atendidos em um laboratório de análises clínicas no município de Juazeiro do Norte- CE. Revista Interfaces - Saúde, Humanas e Tecnologia, 3, 6-9.

Santos, S. A. \& Merlini, L. S. (2010). Prevalência de enteroparasitoses na população do município de Maria Helena, Paraná. Ciência \& Saúde Coletiva, $15,899-905$.

Souza, A. I. et al. (2002). Enteroparasitoses, anemia e estado nutricional em grávidas atendidas em serviço público de saúde. Revista Brasileira Ginecologia, 24, 9-253.

Souza, E. B. (2010). Transição nutricional no Brasil: análise dos principais fatores. Cadernos UniFOA, 5, 49-53.

Stoltzfus, R. J. \& Dreyfuss, M. L. (2013). Prevention of Iron Deficiency Anaemia in Adolescents Role of Weekly Iron Acid Supplementation. Pediatrics, 138(46).

Walcher, D. L. \& Frizzo, M. N. (2013) Associação entre parasitoses intestinais e alterações do hemograma. Revista Mirante - FACOS/CNEC, 3, 18-40.

Whaley, S. E. et al. (2003). Animal Source Foods to Improve Micronutrient Nutrition and Human Function in Developing Countries. The Journal of Nutrition, 133 (39), 65-71.

World Health Organization. (2001). In: Prevention and Control of Iron Deficiency Anaemia in Women and Children. Geneva, Switzerland.

Young, J. et al. (2013). Novel aspects of health promoting compounds in meat. Meat Science, 95, 904-911. 